\title{
Phase Separation and Crystallization Behavior in Extruded Polypropylene/Ethylene-Propylene Rubber Blends Containing Ethylene- $a$-Olefin Copolymers
}

\author{
Joo Hyung LEE, Jong Kwan LEe, Kwang Hee LeE, ${ }^{\dagger}$ and Chang Hyung LEE* \\ Center for Advanced Functional Polymers, \\ School of Chemical Science and Engineering, Inha University, Inchon 402-751, Korea \\ * Medical Devices and Radiation Health Department, \\ Korea Food and Drug Administration, Seoul 122-020, Korea
}

(Received June 21, 1999)

\begin{abstract}
Liquid-liquid (L-L) phase separation and its effects on crystallization in polypropylene (PP)/ethylenepropylene rubber (EPR)/ethylene- $\alpha$-olefin copolymer (ECP) ternary blends obtained by melt extrusion were investigated by time-resolved light scattering (TR-LS) and optical microscopy. L-L phase separation via spinodal decomposition (SD) was confirmed by TR-LS data. After $\mathrm{L}-\mathrm{L}$ phase-separation at $250^{\circ} \mathrm{C}$ for various durations, the blend sample was subjected to temperature-drop to $130^{\circ} \mathrm{C}$ for isothermal crystallization and the effects of $\mathrm{L}-\mathrm{L}$ phase separation on crystallization were investigated. Memory of $\mathrm{L}-\mathrm{L}$ phase separation via SD remained for crystallization. The crystallization rate decreased with increasing $\mathrm{L}-\mathrm{L}$ phase-separated time at $250^{\circ} \mathrm{C}$. Slow crystallization for the long $\mathrm{L}-\mathrm{L}$ phase-separated time could be ascribed to decrease in chain mobility of PP by decrease in rubbery components in the PP-rich region. The ethylene-octene copolymer exhibited good affinity with PP, leading to a slow growth of a concentration fluctuation during annealing.
\end{abstract}

KEY WORDS Polypropylene / Ethylene-Propylene Rubber / Ethylene- $\alpha$-Olefin Copolymer / Phase Separation / Crystallization /

Polypropylene/ethylene-propylene rubber (PP/EPR) blends are injection-moldable thermoplastics with wide applications for automobile parts, appliances and other industrial uses because of good mechanical properties and processability. To further improve properties and expand applications, a new PP/EPR blend containing ethylene- $\alpha$-olefin copolymers (ECP) has been recently developed. ${ }^{1}$

In PP/EPR/ECP ternary blends obtained by melt extrusion, liquid-liquid $(\mathrm{L}-\mathrm{L})$ phase separation at high temperatures is very similar to the spinodal decomposition (SD) which should originate from the partially miscible system. When the blend has lower critical solution temperature (LCST) or the upper critical solution temperature (UCST) phase diagram, the formation of crystalline structures should be affected by L-L phase separation. ${ }^{2,3}$ When LCST is located above the melting point of $\mathrm{PP}, \mathrm{L}-\mathrm{L}$ phase separation precedes crystallization and has significant influence on crystallization. For better control of the physical properties of materials, we need more information on phase behavior and effects on crystallization and thus investigated the phase separation and crystallization behavior in extruded PP/EPR/ECP blends.

We first carried out time-resolved light scattering (TRLS) studies to confirm $\mathrm{L}-\mathrm{L}$ phase separation via $\mathrm{SD}$ in PP/EPR/ECP blends and investigated the effects of $\mathrm{L}-\mathrm{L}$ phase separation on crystallization.

\section{EXPERIMENTAL}

\section{Materials}

An isotactic PP was supplied by LG Chemical Co. The density was $0.908 \mathrm{~g} \mathrm{~cm}^{-3}$ and the melt flow rate (MFR) measured by ASTM D1238L was 60. EPR was supplied by SK Co. The EPR contained $54 \mathrm{~mol} \%$ ethylene and the Mooney viscosity, $\mathrm{ML}_{1+4}\left(125^{\circ} \mathrm{C}\right)$ measured by ASTM D 1646 was 14. Two commercial ECPs were used. ECP-1 was an ethylene-octene copolymer with density of 0.885 $\mathrm{g} \mathrm{cm}^{-3}$ and a 1.0 MFR measured by ASTM D1238E (Dow Chemical Co., Engage 8003; octene content = 18\%). ECP-2 was an ethylene-butene copolymer with density of $0.880 \mathrm{~g} \mathrm{~cm}^{-3}$ and a $0.8 \mathrm{MFR}$ (Exxon Chemical Co., Exact 4033).

$\mathrm{PP} / \mathrm{EPR} / \mathrm{ECP}$ ternary blends were prepared in a twostage process. EPR/ECP binary blends made in a Farrel Banbury BR type mixer was subsequently melt-mixed with $\mathrm{PP}$ at $250^{\circ} \mathrm{C}$ on a $30 \mathrm{~mm}$ co-rotating twin screw extruder (Werner Pfleiderer) at a speed of 200 RPM. The screw configuration was basic kneading blocks, followed by gear mixer flights, to produce a medium shear and high mixing configuration. In each run, the extrudate was quickly quenched in ice-water to freeze the structure in the melt and then was chopped into granules. The composition of the PP/EPR/ECP blends was 60/30/ 10 by weight.

\section{$T R-L S$ and $O M$}

A thin film specimen (ca. $15 \mu \mathrm{m}$ thick) was prepared by pressing the blend pellets between two cover glasses at $250^{\circ} \mathrm{C}$. Immediately after the melt-pressing, the specimen was quickly transferred onto a hot stage in a light scattering photometer equipped with a CCD (chargecoupled device) camera ${ }^{4}$ and the kinetics of $\mathrm{L}-\mathrm{L}$ phase separation were investigated.

After the $\mathrm{L}-\mathrm{L}$ phase-separation at $250^{\circ} \mathrm{C}$ for a certain

\footnotetext{
${ }^{\dagger}$ To whom correspondence should be addressed (E-mail : polylee@inha.ac.kr).
} 
(a)

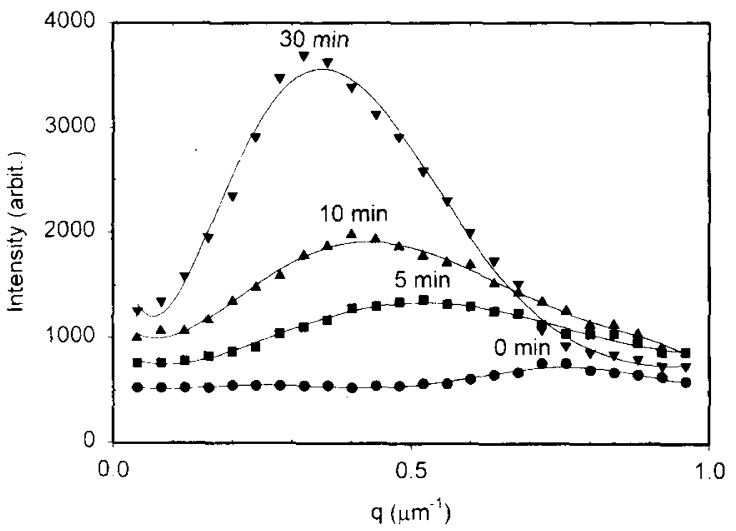

(b)

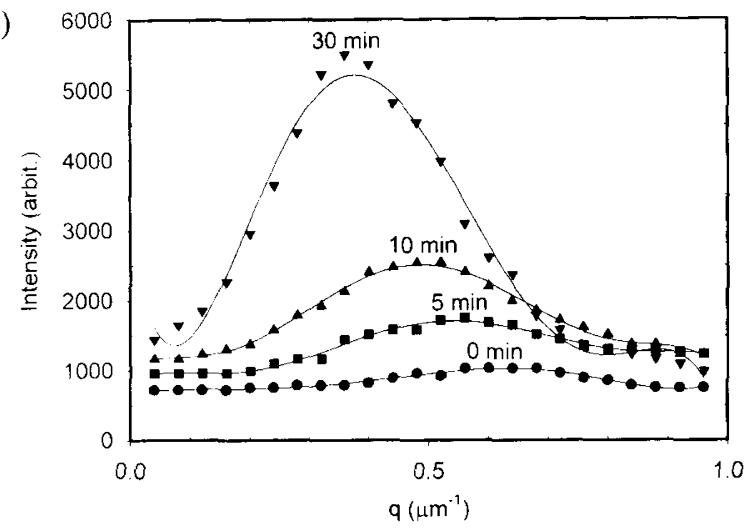

Figure 1. Changes in light scattering profiles of PP/EPR/ECP blends during $\mathrm{L}-\mathrm{L}$ phase separation at $250^{\circ} \mathrm{C}$ : (a) PP/EPR/ECP-1 blend ; (b) PP/EPR/ECP-2 blend.

time $\left(t_{\mathrm{s}}\right)$, the sample was rapidly transferred onto a light scattering hot stage set at $130^{\circ} \mathrm{C}$ for isothermal crystallization and the effects of $\mathrm{L}-\mathrm{L}$ phase separation on crystallization were investigated. A polarized He-Ne gas laser of $632.8 \mathrm{~nm}$ wavelength was applied to the film specimen. $V_{v}$ geometry, in which the optical axis of the analyzer was set parallel to that of the polarizer, was used.

The final morphology of the crystallized specimen at $130^{\circ} \mathrm{C}$ was observed under an optical microscope (OM).

\section{DMTA}

The pellets were compression-molded between metal plates at $250^{\circ} \mathrm{C}$ for $3 \mathrm{~min}$. After molding, the specimen was rapidly transferred into an oil bath set at $130^{\circ} \mathrm{C}$ and was crystallized. This crystallized specimen was used for dynamic mechanical measurements performed with a Polymer Laboratories dynamic mechanical thermal analyzer (DMTA) MK III. DMTA was operated in the dual cantilevers bending mode at a frequence of $1 \mathrm{~Hz}$ and at a heating rate of $3^{\circ} \mathrm{C} \mathrm{min}-1$ in a temperature range of from -120 to $80^{\circ} \mathrm{C}$.

\section{RESULTS AND DISCUSSION}

\section{L-L Phase Separation Behavior}

Figure 1 shows changes of the one-dimensional $V_{v}$ scattering profiles with $\mathrm{L}-\mathrm{L}$ phase separation time, $t_{\mathrm{s}}$ in which the scattering vector $q$ is defined by $q=\left(4 \pi / \lambda^{\prime}\right)$ $\sin (\theta / 2)$, where $\lambda^{\prime}$ is the wavelength of light in the speci- (a)

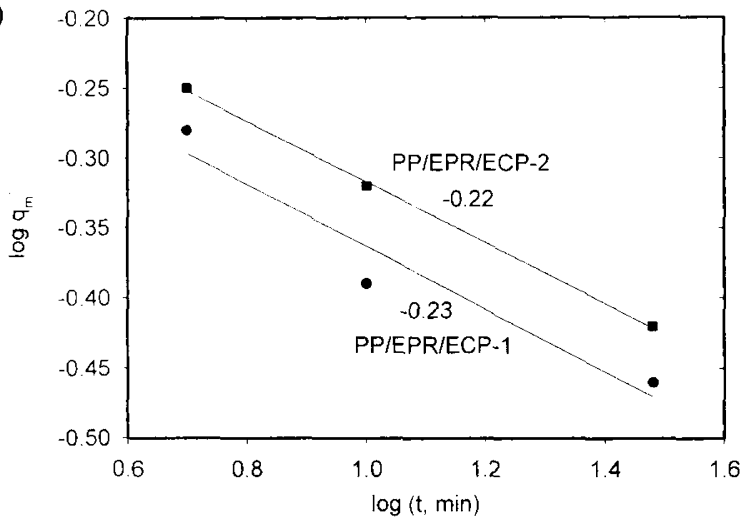

(b)

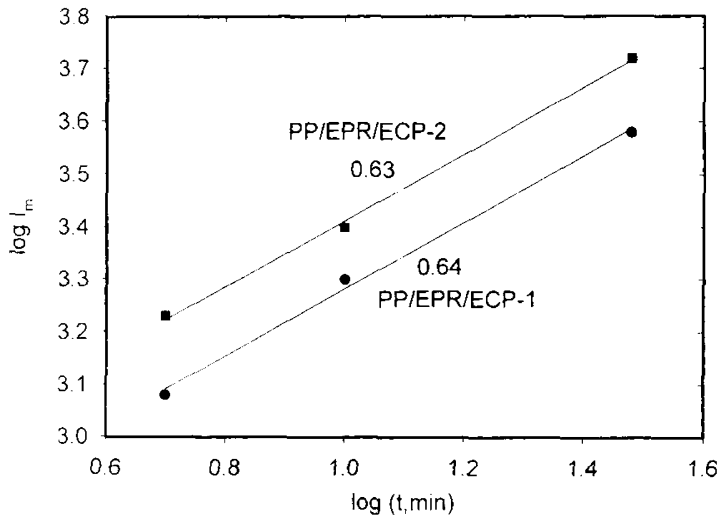

Figure 2. $\log -\log$ plots of (a) $q_{\mathrm{m}}(t) \propto t^{-\alpha}$ and (b) $I_{\mathrm{m}}(t) \propto t^{\beta}$.

men and $\theta$ is the scattering angle. Even at $t_{\mathrm{s}}=0$, a weak scattering peak appears at large $q$, suggesting an appreciable development of $\mathrm{L}-\mathrm{L}$ phase separation in the quenched-and-remelt specimen. The peak intensity $I_{\mathrm{m}}$ increases with $t_{s}$, and its position $q_{m}$ shifts to smaller angles, implying that the $\mathrm{L}-\mathrm{L}$ phase separation proceeds via SD mechanism.

The phase separation process seen in Figure 1 can be interpreted by the power law relationship. This problem is discussed by Langer based on non-linear statistical consideration. ${ }^{5}$ The theory predicts the power law scheme for the intermediate-to-late stages of SD as

$$
\begin{aligned}
& q_{\mathrm{m}}(t) \propto t^{-\alpha} \\
& I_{\mathrm{m}}(t) \propto t^{\beta}
\end{aligned}
$$

where $\alpha$ and $\beta$ are the critical exponents. Figures $2 \mathrm{a}$ and $2 \mathrm{~b}$ show the $\log -\log$ plots of $q_{\mathrm{m}}$ and $I_{\mathrm{m}}$ versus phase separation time, respectively. We see that the $\beta / \alpha$ ratio is approximately 3 , which is in good accord with the $\mathrm{dy}$ namics of cluster coalescence, suggested by Binder and Stauffer ${ }^{6}$ for the late stage of SD or by Siggia ${ }^{7}$ for the intermediate stage.

In Figure 3, the periodic length $\Lambda_{\mathrm{m}}$ obtained by applying the Bragg equation to the peak position of the light scattering profiles is plotted as a function of $t_{\mathrm{s}}$. Increase of $\Lambda_{\mathrm{m}}$ with $t_{\mathrm{s}}$ can be clearly seen. $\Lambda_{\mathrm{m}}$ of the PP/EPR/ ECP-1 system is smaller than that of the PP/EPR/ECP-2 system, suggesting the slower growth of SD. The ethylene-octene copolymer (ECP-1) may exhibit better solubility with PP and EPR at high shear fields, leading to a slower $\mathrm{L}-\mathrm{L}$ demixing in quiescent state.

Dynamic mechanical measurements provide further 


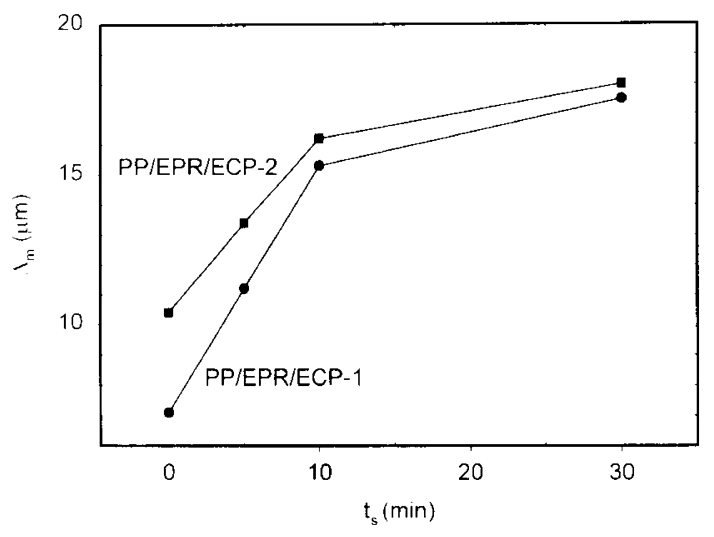

Figure 3. Change of $\Lambda_{\mathrm{m}}$ with $t_{\mathrm{s}}$.

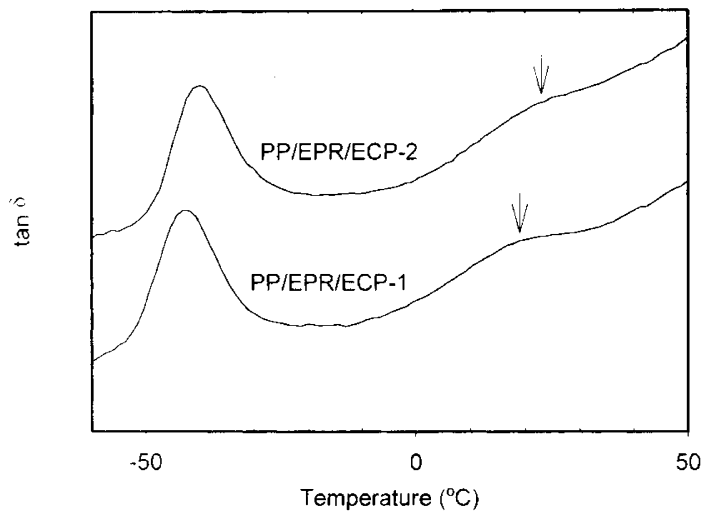

Figure 4. Temperature dependence of loss tangent $(\tan \delta)$ for PP/ EPR/ECP blends.

evidence for the slower demixing. The mechanical loss tangent, $\tan \delta$ of the PP/EPR/ECP blends is plotted in Figure 4 as a function of temperature. Both blends show two distinct glass transitions, suggesting phaseseparated morphology. The low and high glass transition peaks correspond to the glass transition temperature, $T_{\mathrm{g}}$ of rubber region and $T_{\mathrm{g}}$ of PP-rich region (the $T_{\mathrm{g}} \mathrm{s}$ of pure PP, EPR, ECP-1, and ECP-2 were 28.5, -38.6, - 56.4, and $-50.2^{\circ} \mathrm{C}$, respectively). The high $T_{\mathrm{g}}$ of the PP/EPR/ ECP-1 blend is lower than that of the PP/EPR/ECP-2 blend. This suggests the less segregation of rubber components (EPR and ECP) from PP-rich region in the ECP1 system than in the ECP-2 system (a relatively larger amount of remaining rubbery components in the PP-rich region).

Supplemental evidence of $\mathrm{L}-\mathrm{L}$ phase separation was provided through observation of structure formation at $130^{\circ} \mathrm{C}$. The apparent mutual diffusion coefficient $D_{\text {app }}$ at $130^{\circ} \mathrm{C}$ was very low. ${ }^{8}$ This implies that the L-L phase separation rate may be negligible at $130^{\circ} \mathrm{C}$. The crystallization rate of $\mathrm{PP}$ is very high (crystallization completed in about $10 \mathrm{~s}$, as discussed in Figure 9). In such case, the $\mathrm{L}-\mathrm{L}$ phase separation morphology, i.e., the periodic and interconnected structure, could be preserved during crystallization. $^{2}$ Figures 5 and 6 show optical micrographs of the PP/EPR/ECP blends crystallized at 130 ${ }^{\circ} \mathrm{C}$ after the $\mathrm{L}-\mathrm{L}$ phase separation at $250^{\circ} \mathrm{C}$. A dramatic change in the morphology of the blends with annealing at $250^{\circ} \mathrm{C}$ is observed. At the beginning [Figures $5 \mathrm{a}$ and
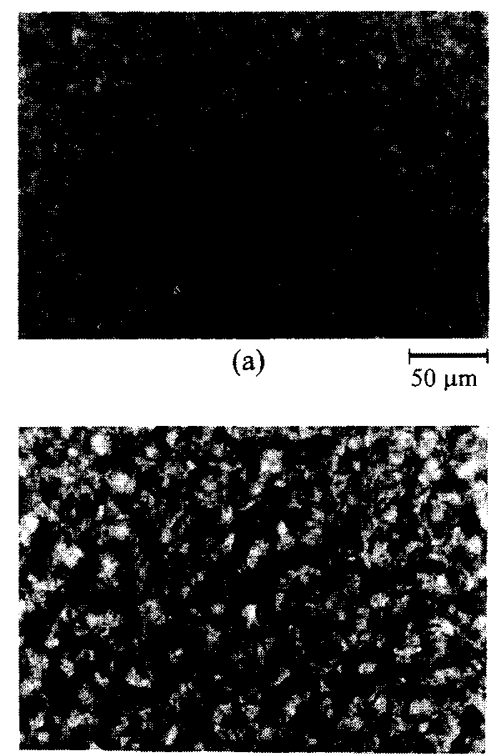

(b)

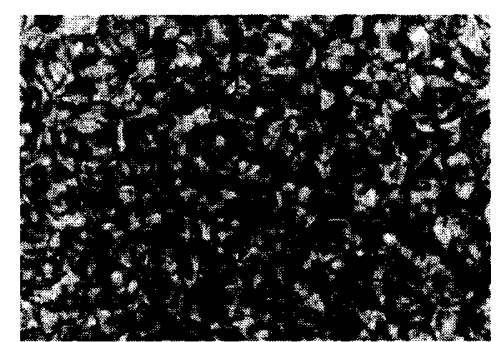

(c)

Figure 5. Optical micrographs of PP/EPR/ECP-1 blend crystallized at $130^{\circ} \mathrm{C}$ for $10 \mathrm{~min}$ after the $\mathrm{L}-\mathrm{L}$ phase separation at $250^{\circ} \mathrm{C}$ for (a) $t_{\mathrm{s}}=0 \mathrm{~min}$; (b) $t_{\mathrm{s}}=10 \mathrm{~min}$; (c) $t_{\mathrm{s}}=30 \mathrm{~min}$.

$6 \mathrm{a}$ ], it is not clear which phase is continuous and which is dispersed. At the later stages of $\mathrm{L}-\mathrm{L}$ phase separation, phase connectivity grows and eventually breaks up into macroscopic spherical texture. These are characteristic to the SD mechanism. The spacing between dark and bright regions in the micrographs was in good agreement with $\Lambda_{\mathrm{m}}$ obtained from the light scattering profiles.

Based on the above results, a scenario of the melt extrusion to yield near a homogeneous blend may be given as follows : at the high shear rates in the exturder, the spinodal temperature $T_{\mathrm{s}}$ in LCST phase diagram may be elevated, as shown by the arrow in Figure 7 and onephase region becomes wider. ${ }^{9-12}$ Thus, the system undergoes phase dissolution. The dissolution continues until a new composition is reached in the mixture. The compositions, $X_{\mathrm{A}}$ and $X_{\mathrm{B}}$, at a static condition move to the compositions, $X_{\mathrm{A}}{ }^{\prime}$ and $X_{\mathrm{B}}{ }^{\prime}$ in the shear field. This could be the case for the present PP/EPR/ECP blends. If $T_{\mathrm{s}}$ in the shear field is raised above the barrel temperature $T_{\mathrm{b}}$, the mixing can be done in one-phase region to obtain a homogeneous mixture. The phase diagram in Figure 7 is just a schematic illustration and does not have any quantitative significance ; it indicates that the mixture was thermodynamically unstable. It is conceivable that the blend may not have a LCST phase diagram but rather has an UCST phase diagram. In that case, $T_{\mathrm{s}}$ in the shear fields should be lowered to induce the disso- 


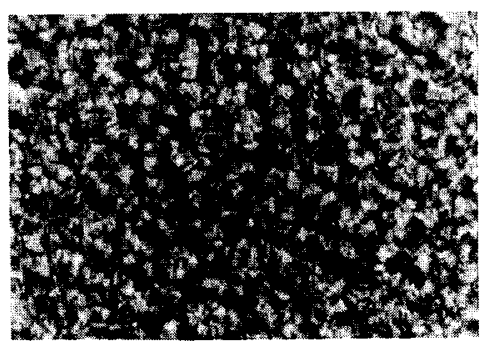

(a)

$\stackrel{\longrightarrow}{50 \mu \mathrm{m}}$

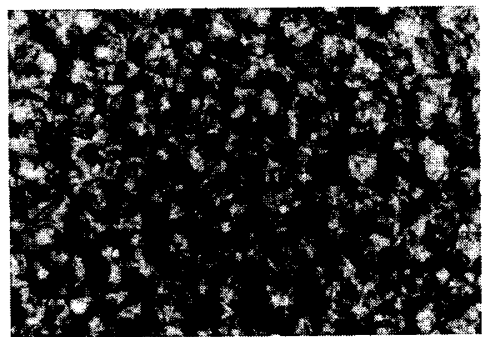

(b)

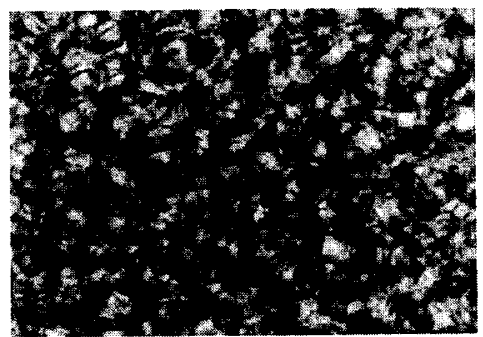

(c)

Figure 6. Optical micrographs of PP/EPR/ECP-2 blend crystallized at $130^{\circ} \mathrm{C}$ for $10 \mathrm{~min}$ after the $\mathrm{L}-\mathrm{L}$ phase separation at $250^{\circ} \mathrm{C}$ for (a) $t_{\mathrm{s}}=0 \mathrm{~min}$; (b) $t_{\mathrm{s}}=10 \mathrm{~min}$; (c) $t_{\mathrm{s}}=30 \mathrm{~min}$.

lution of the components. Once the melt is extruded out from the nozzle, the shear rate turns zero and $T_{\mathrm{s}}$ immediately falls to a static value so that the SD proceeds until the structure is fixed by crystallization.

\section{Effects of $L-L$ Phase Separation on Crystallization}

$\mathrm{L}-\mathrm{L}$ phase-separated specimens at $250^{\circ} \mathrm{C}$ for various times were allowed to crystallize by rapid temperature drop to $130^{\circ} \mathrm{C}$ for the isothermal crystallization.

Figure 8 shows typical change in the $V_{v}$ scattering profiles during crystallization at $130^{\circ} \mathrm{C}$. The peak position, indicative of $\Lambda_{\mathrm{m}}$ caused by SD at $250^{\circ} \mathrm{C}$, is kept constant and its intensity $\left(I_{\mathrm{m}}\right)$ increases with time. Constant $\Lambda_{\mathrm{m}}$ suggests that $\mathrm{L}-\mathrm{L}$ phase separation no longer proceeds during crystallization. The increase of $I_{\mathrm{m}}$ should be caused by crystallization. The crystallization increases the refractive index of PP-rich region, causing larger optical contrast in phase-separated system.

To discuss crystallization, it is convenient to use the light scattering invariant in the $V_{v}$ mode, $Q_{V_{v}}$. The time variation of $Q_{V_{v}}$ is shown in Figure 9. $Q_{V_{v}}$ is kept constant for a while, increases rapidly and attains a maximum value. Then, $Q_{V_{v}}$ decreases slightly and levels off. Finite $Q_{V_{v}}$ at time zero may be ascribed to the density fluctuation formed by SD at $250^{\circ} \mathrm{C}$ before crystallization onset. The constant $Q_{V_{v}}$ at early time is indicative of the incubation period typical of the nucleation process of the crystallization. The rapid increase of $Q_{V_{v}}$ should be as-

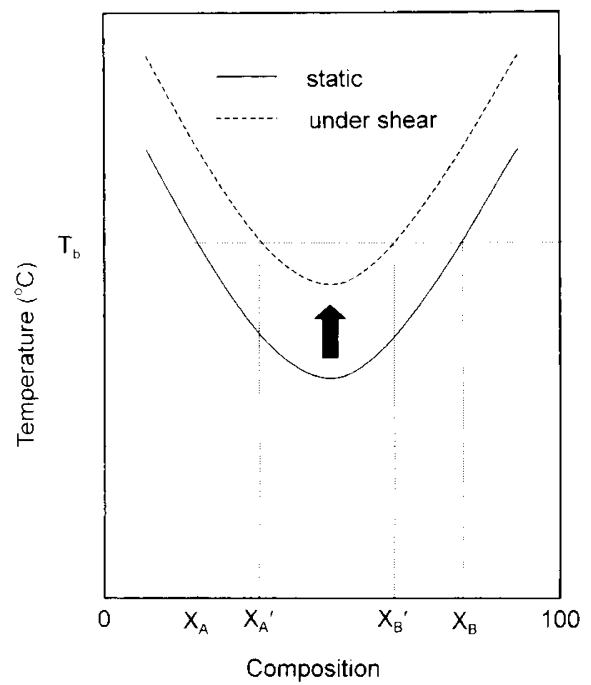

Figure 7. Schematic drawing of changes in phase diagram with shear rate. Arrow indicates spinodal temperature to elevate at high shear rate. $T_{\mathrm{b}}$ is the barrel temperature in an extruder.

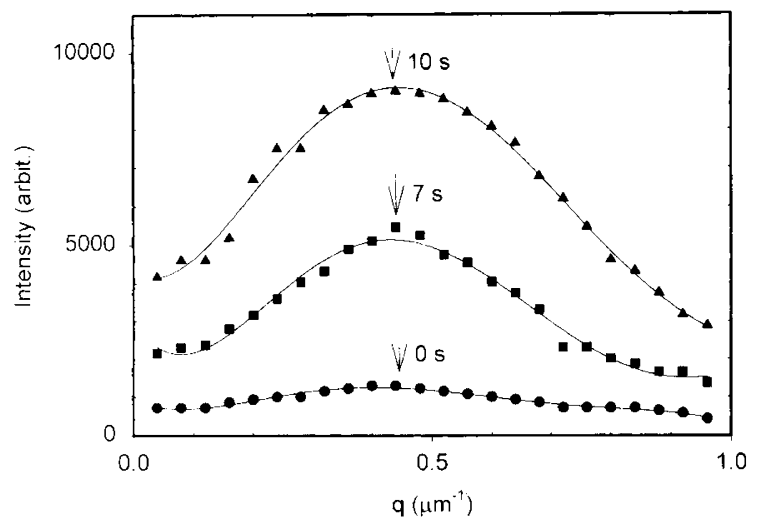

Figure 8. Change in light scattering profile of PP/EPR/ECP-1 blend during crystallization at $130^{\circ} \mathrm{C}$ after $t_{\mathrm{s}}=10 \mathrm{~min}$.

cribed to PP crystallization. The slight decrease and leveling-off of $Q_{V_{v}}$ suggest that the $\mathrm{PP}$ in the rubber-rich region may crystallize slowly at the later stages, reducing differences in refractive index between the separated regions.

Neglecting the small contribution from optical anisotropy, $Q_{V_{v}}$ of crystallized polymer systems can be approximated by ${ }^{13}$

$$
Q_{V_{v}} \propto \phi_{c}\left(1-\phi_{c}\right)\left(\alpha_{c}-\alpha_{0}\right)
$$

where $\phi_{\mathrm{c}}$ is the volume fraction of crystalline aggregate, $\alpha_{\mathrm{c}}$ and $\alpha_{0}$ is the polarizability of crystalline aggregate and the melt, respectively. At the early stage of crystallization, eq 3 is further approximated by

$$
Q_{V_{\mathrm{v}}} \propto \phi_{\mathrm{c}}\left(\alpha_{\mathrm{c}}-\alpha_{0}\right)
$$

$Q_{V_{v}}$ is assumed to be proportional to $\phi_{\mathrm{c}}$ so that the linear growth rate of crystallite $G$ is,

$$
G \propto \mathrm{d}\left(Q_{V_{\mathrm{v}}}\right)^{1 / 3} / \mathrm{d} t
$$

Therefore, one can estimate $G$ from the initial slope of the time variation of $\left(Q_{V_{\mathrm{v}}}\right)^{1 / 3}$. $G$, estimated by eq 5 , is shown as a function of $t_{\mathrm{s}}$ in Figure 10. $G$ decreases with 

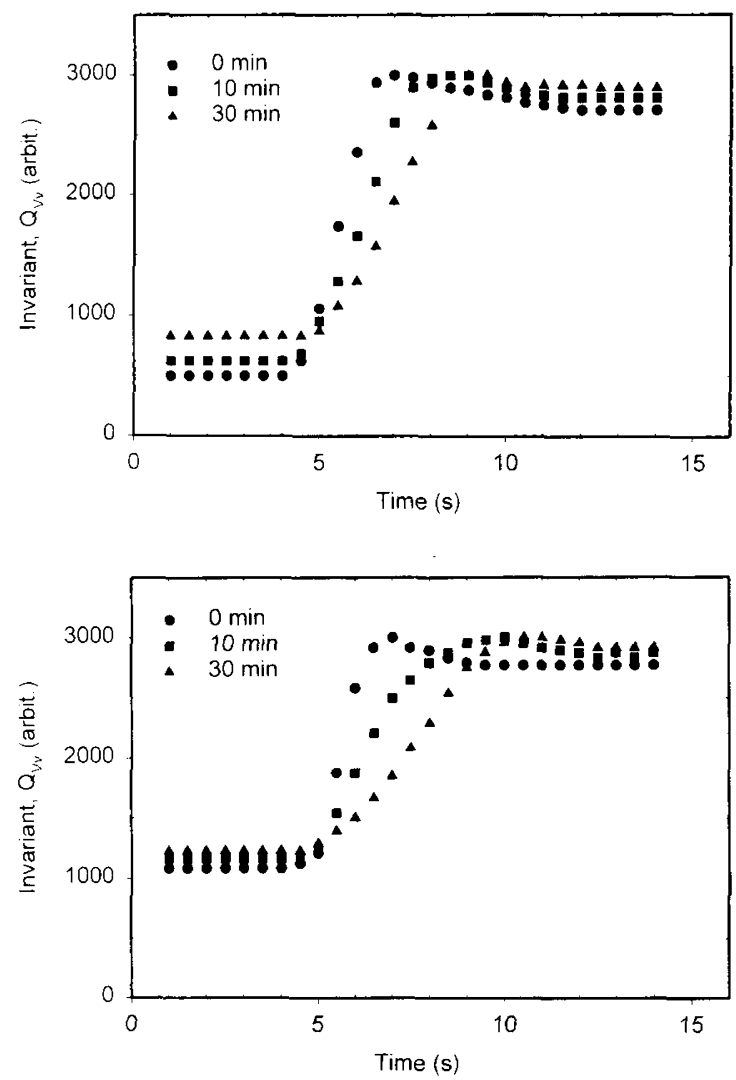

Figure 9. Time variation of the invariant $Q_{\mathrm{K}}$ in PP/EPR/ECP blends crystallized at $130^{\circ} \mathrm{C}$ after $\mathrm{L}-\mathrm{L}$ phase separation at $250^{\circ} \mathrm{C}$ for $t_{\mathrm{s}}=0,10$ and $30 \mathrm{~min}$ : (a) PP/EPR/ECP-1 blend ; (b) PP/EPR/ ECP-2 blend.

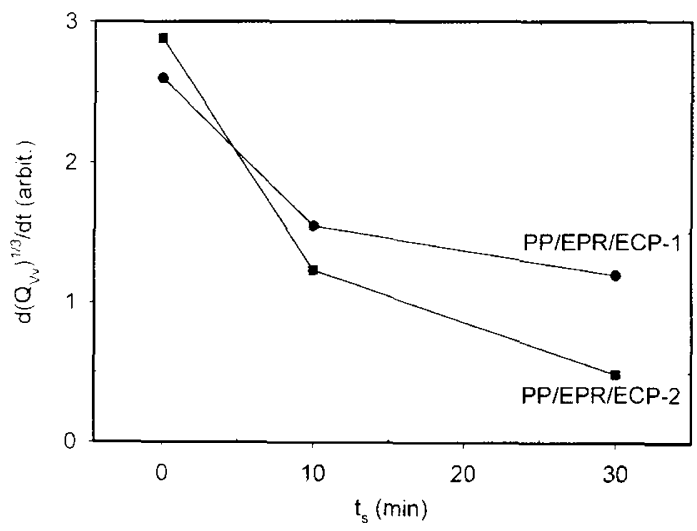

Figure 10. Change in $G$ with $t_{\mathrm{s}}$.

$t_{\mathrm{s}}$; for $G$ for $t_{\mathrm{s}}=10$ and $30 \mathrm{~min}$ is much smaller than that for $t_{\mathrm{s}}=0 \mathrm{~min}$. This may come from differences of amounts of rubbery components (EPR and/or ECP) in PP-rich region. The growth of concentration fluctuation is realized by up-hill diffusion; A molecules diffuse into A-rich region from B-rich region. Thus, in PP/EPR/ECP blends, EPR and ECP molecules are forced to move from PP-rich region to rubber-rich region. As the $\mathrm{L}-\mathrm{L}$ phase separation proceeds, the amounts of rubbery compo- nents in PP-rich region should decrease by SD. In PPrich region, the $T_{\mathrm{g}}$ increases as the amount of the rubbery components decreases. The crystallization rate consists of nucleation and diffusion rates. The higher $T_{\mathrm{g}}$ reduces the diffusion rate so that the crystallization rate may be correspondingly reduced.

Figure 10 shows that the dependence of $G$ on $t_{\mathrm{s}}$ is noticeably influenced by the type of ECP. Stronger influence is observed for the PP/EPR/ECP-2 blend containing ethylene-butene copolymer. This can be explained by differences in the growth rate of the concentration fluctuation; i.e., since concentration fluctuation by SD occurred more rapidly in the PP/EPR/ECP-2 blend, the larger amounts of rubbery components in PP-rich phases decreased with $t_{s}$, and the decrease may retard crystallization.

\section{CONCLUSION}

L- $\mathrm{L}$ phase separation via $\mathrm{SD}$ was found in extruded PP/EPR/ECP blends. For homogeneity of melt-extruded blends, one may explain that the phase diagram is elevated at high shear rates in the extruder, allowing phase dissolution. The effects of $\mathrm{L}-\mathrm{L}$ phase separation on the crystalline morphology produced by subsequent crystallization are discussed. Crystallization takes place only in the PP-rich region so that the memory of SD (the periodic structure) is preserved. The amounts of rubbery components in PP-rich region formed by SD were strongly related to the crystallization rate. The amounts of the rubbery components in PP-rich region decreased with increasing the $\mathrm{L}-\mathrm{L}$ phase separation time and the decrease retarded the crystallization rate. The affinity of ethylene-octene copolymer with PP may be higher than that of ethylene-butene copolymer.

Acknowledgments. This research was supported by the Center for Advanced Functional Polymers.

\section{REFERENCES}

1. T. Nomura, T. Nishino, K. Iwanami, K. Yokomizo, K. Kitano, and S. Toki, J. Appl. Polym. Sci., 55, 1307 (1995).

2. N. Inaba, K. Sato, S. Suzuki, and T. Hashimoto, Macromolecules, 19, 1690 (1986).

3. N. Inaba, T. Yamada, S. Suzuki, and T. Hashimoto, Macromolecules, 21, 407 (1988).

4. C. H. Lee, H. Saito, and T. Inoue, Macromolecules, 28, 8096 (1995).

5. J. S. Langer, M. Baron, and H. S. Miller, Phys. Rev. (A), 11, 1417 (1975).

6. K. Binder and D. Stauffer, Phys. Rev. Lett., 33, 1006 (1974).

7. E. D. Siggia, Phy. Rev. (A), 20, 595 (1979).

8. S. W. Im, K. H. Lee, and C. H. Lee, Polymer, 40, 2837 (1999).

9. I. Hindawi, J. S. Higgins, A. F. Galambos, and R. A. Weiss, Macromolecules, 23, 670 (1990).

10. M. Okamoto and T. Inoue, Polymer, 35, 257 (1994).

11. M. Okamoto, K. Shiomi, and T. Inoue, Polymer, 36, 87 (1995).

12. M. Okamoto and T. Kotaka, Polymer, 38, 1357 (1997).

13. J. T. Koberstein, T. P. Russel, and R. S. Stein, J. Polym. Sci., Polym. Phys., 17, 1719 (1979). 